\title{
Model on Ecological Risk Assessment, Prediction and Early Warning of Land Use in Shengjin Lake Nature Reserve
}

chenbin zhang $^{1}$, bin dong ${ }^{2}$, lili feng ${ }^{1}$, shuangshuang zhang ${ }^{1}$, yuting wang ${ }^{1}$, sheng li $^{1}$, and jian chen $^{1}$

${ }^{1}$ Affiliation not available

${ }^{2}$ Anhui Agricultural University

May 25, 2020

\begin{abstract}
With reference to 1986,1995,2004,2011 and 2016 TM images of the Shengjin Lake National Nature Reserve in Anhui province, this paper used ERDAS and ARCGIS software to collect the different land use information on the experimental, buffer and core areas for more than 30 years. By combining the social and economic data and selecting the index which is about the ecological risk of land use to built the system of land utilization evaluation index on ecological risk. On this basis, the AHP and expert commonly were used to determine the weight of index and establish the assessment model on ecological risk of the land use by using the method of fuzzy mathematics. With reference to the ecological risk index which was calculated, and adopting the interpolation and three regression methods to built a forecasting model .Finally, the computer simulation was used to establish the early warning for ecological risk of the land use.
\end{abstract}

\section{Hosted file}

3.doc available at https://authorea.com/users/325916/articles/453894-model-on-ecologicalrisk-assessment-prediction-and-early-warning-of-land-use-in-shengjin-lake-nature-reserve 